\title{
SCALING OF HIGH-QUANTILE ESTIMATORS
}

\author{
MATTHIAS DEGEN, ${ }^{*} * *$ ETH Zürich \\ PAUL EMBRECHTS, ${ }^{* * *}$ ETH Zürich and Swiss Finance Institute
}

\begin{abstract}
Enhanced by the global financial crisis, the discussion about an accurate estimation of regulatory (risk) capital a financial institution needs to hold in order to safeguard against unexpected losses has become highly relevant again. The presence of heavy tails in combination with small sample sizes turns estimation at such extreme quantile levels into an inherently difficult statistical issue. We discuss some of the problems and pitfalls that may arise. In particular, based on the framework of second-order extended regular variation, we compare different high-quantile estimators and propose methods for the improvement of standard methods by focusing on the concept of penultimate approximations.
\end{abstract}

Keywords: Extreme value theory; peaks over threshold; penultimate approximation; power normalization; second-order extended regular variation

2010 Mathematics Subject Classification: Primary 60G70

Secondary $62 \mathrm{G} 32$

\section{Introduction}

It is fair to say that the global financial system is going through a deep crisis. Whereas for some time a regulatory framework was put into place to avoid systemic risk, the current problems highlight the total insufficiency of this (so-called) Basel framework. Warnings for this were voiced early on; see, for instance, [9]. Also, the weaknesses of value-at-risk (VaR), the risk measure required by the Basel framework, were discussed over and over again; see, for instance, [21] and the references therein. Nevertheless, it has turned out to be extremely difficult to convince regulators to 'think again'. As a consequence, and mainly spurred on by the subprime crisis, statisticians are increasingly called upon to single out research themes with considerable practical usefulness. A key example of this is the long-term joint project between the Office of the Comptroller of the Currency (OCC) and the National Institute of Statistical Sciences (NISS) on the topic of 'Financial Risk Modeling and Banking Regulation'. The current paper is motivated by this research program.

Our starting point is the discussion about the estimation of regulatory (risk) capital a financial institution needs to hold in order to safeguard against unexpected losses. Without going into a full description of financial data-be it market risk (MR), credit risk (CR), or operational risk (OR) - it suffices to know that, according to the current regulatory standards in the banking industry (Basel II/III framework), risk capital has to be calculated (statistically estimated) using the concept of VaR at very high levels of confidence (for MR, usually $99 \%$ at a 10-day horizon; for CR and OR, $99.9 \%$ and, for economic capital, $99.97 \%$, all three at a one-year horizon).

Received 23 March 2009; revision received 19 June 2011.

* Postal address: Department of Mathematics, ETH Zürich, Raemistrasse 101, CH-8092 Zürich, Switzerland.

** Email address: degen@math.ethz.ch

*** Email address: embrechts@math.ethz.ch 
The credit crisis prompted the introduction of an extra $99.9 \%$, one-year capital charge for MR, the so-called incremental risk charge; see Basel Committee [3]. Because of the extreme quantile levels required, early on extreme value theory (EVT) was recognized as a potentially useful tool. However, and this often from practice, critical voices have been raised against an imprudent use of (standard) EVT. In the context of quantitative risk management (QRM), the use of EVT-based high-quantile estimators may indeed be a delicate issue and warrants careful further study.

The aim of our paper is twofold. In the first and more theoretical part, we analyze different choices of normalization and their influence on the rate of convergence in certain limit laws underlying EVT. In the second part, concrete applications of the methodology developed in the first part are discussed.

The paper is organized as follows. In Section 2 we introduce some basic concepts from EVT. In Section 3 we discuss the concept of normalized high-risk scenarios and, in Section 4 , compare the effects of linear versus power norming for high-risk scenarios and quantiles using the framework of first- and second-order extended regular variation. Based on the findings from these asymptotic results, we propose the use of so-called penultimate approximations to estimate extreme quantiles. In Section 5 we compare the performance of different high-quantile estimators. One method increasingly championed in practice estimates quantiles at a lower level (e.g. 99\%) and then scales up to the desired higher level (e.g. 99.9\%) according to some scaling procedure to be specified. In this context, the usefulness of penultimate approximations in situations of very heavy tails together with small sample sizes (typical for OR) is highlighted.

\section{Univariate EVT}

We assume that the reader is familiar with univariate EVT, as presented for instance in [11] or [13]. Throughout, we assume that our loss data $X>0$ are modeled by a continuous distribution function (DF) $F$ with upper endpoint $x_{F} \leq \infty$ and standardly write $\bar{F}=1-F$. The corresponding tail quantile function is denoted by $U(t)=F^{\leftarrow}(1-1 / t)$, where $F^{\leftarrow}$ denotes the (generalized) inverse of $F$. For properties of $F^{\leftarrow}$, see, for instance, [12]. To avoid confusion, we will — where necessary - denote the DF and the tail quantile function of a random variable (RV) $X$ by $F_{X}$ and $U_{X}$, respectively.

As our focus is on the application of EVT-based methods to quantitative risk management, we prefer to work within the framework of exceedances (the peaks over threshold (POT) method) rather than within the classical framework of block maxima. The two concepts however are closely linked as the next result shows; see [11, Theorem 1.1.6].

Proposition 2.1. For $\xi \in \mathbb{R}$, the following statements are equivalent.

(i) There exist constants $a_{n}>0$ and $b_{n} \in \mathbb{R}$ such that

$$
\lim _{n \rightarrow \infty} F^{n}\left(a_{n} x+b_{n}\right)=H_{\xi}(x)=\exp \left\{-(1+\xi x)^{-1 / \xi}\right\}
$$

for all $x$ with $1+\xi x>0$.

(ii) There exists a measurable function $a(\cdot)>0$ such that, for $x>0$,

$$
\lim _{t \rightarrow \infty} \frac{U(t x)-U(t)}{a(t)}=D_{\xi}(x)=\frac{x^{\xi}-1}{\xi} .
$$


(iii) There exists a measurable function $f(\cdot)>0$ such that

$$
\lim _{t \rightarrow x_{F}} \frac{\bar{F}(t+x f(t))}{\bar{F}(t)}=(1+\xi x)^{-1 / \xi}
$$

for all $x$ for which $1+\xi x>0$.

Moreover, (2.1) holds with $b_{n}=U(n)$ and $a_{n}=a(n)$. Also, (2.3) holds with $f(t)=a(1 / \bar{F}(t))$.

Definition 2.1. A DF $F$ satisfying (2.1) is said to belong to the linear maximum (l-max) domain of attraction of the extreme value distribution $H_{\xi}$ and we write $F \in D_{l}^{\max }\left(H_{\xi}\right)$. For necessary and sufficient conditions for distributions $F$ to belong to $D_{l}^{\max }\left(H_{\xi}\right)$, we refer the reader to [11, Chapter 1].

Domain of attraction conditions have been formulated directly in terms of regular variation of $\bar{F}$ at $x_{F} \leq \infty$ for the cases $\xi>0$ and $\xi<0$, but not for the case $\xi=0$; see [15]. The novelty of Proposition 2.1 (originally due to de Haan [10]) is that it treats the domain of attraction conditions for the three cases in a unified way by making use of the more general concept of extended regular variation (ERV) for $U$. Recall that a function $U$ is said to be of ERV with index $\xi \in \mathbb{R}$ and with auxiliary function $a(\cdot)$ if it satisfies (2.2); see [11, Appendix B.2]. In that case we write $U \in \operatorname{ERV}_{\xi}(a)$.

Remark 2.1. Even within the unified framework of ERV, the case $\xi=0$ is still somewhat special. Acting as limiting cases, the right-hand sides of (2.2) and (2.3) are interpreted as $\log x$ and $\mathrm{e}^{-x}$, respectively. In that case, $U$ and $1 / \bar{F}$ are said to be of $\Pi$-variation and $\Gamma$-variation, respectively, and we write $U \in \Pi(a)$ (or $U \in \mathrm{ERV}_{0}$ ) and $1 / \bar{F} \in \Gamma(f)$.

From a theoretical point of view, this full generality of the framework of ERV is certainly to be appreciated. For applications to QRM however, a framework treating $\xi \geq 0$ but not $\xi<0$ in an as simple as possible way is to be preferred. This is done below by basically working with $\log U$ instead of $U$.

\section{First-order asymptotics of normalized high-risk scenarios and quantiles}

For a positive $\mathrm{RV} X \sim F$, we introduce the notation $X^{t}$, which is defined as the RV $X$, conditioned to exceed the threshold $t>0$. Within QRM, $X^{t}$ is often referred to as a high-risk scenario; see also [1] for this terminology.

With this notation, Proposition 2.1(iii) states that high-risk scenarios, linearly normalized, converge weakly to a nondegenerate limit, i.e. for $\xi \in \mathbb{R}$ and $x>0$,

$$
\mathrm{P}\left(\frac{X^{t}-t}{f(t)}>x\right)=\frac{\bar{F}(t+x f(t))}{\bar{F}(t)} \rightarrow-\log H_{\xi}(x)=(1+\xi x)^{-1 / \xi} \quad \text { as } t \rightarrow x_{F}
$$

for some measurable function $f(\cdot)>0$. In this case we will say that $F$ belongs to the linear POT (l-POT) domain of attraction of $H_{\xi}$ and write $F \in D_{1}^{\mathrm{POT}}\left(H_{\xi}\right)$.

While the limit behavior of random variables (exceedances as well as block maxima) under linear normalizations is well understood and frequently used in applications, the theory under nonlinear normalizations has been studied less. Pantcheva [22] and Mohan and Ravi [19] developed a theory of power norming within the block-maxima framework.

We will adopt this idea of nonlinear norming and study the limit behavior of power normalized high-risk scenarios. Inspired by Barakat et al. [2], who compared the convergence rates under 
linear and power normalizations within the block-maxima setting, we study the first- and secondorder asymptotic behaviors of power-normalized high-risk scenarios and quantiles.

Definition 3.1. We say that a DF $F$ belongs to the power POT ( $p$-POT) domain of attraction of some nondegenerate DF $K$ and write $F \in D_{\mathrm{p}}^{\mathrm{POT}}(K)$, if there exists a measurable function $g(\cdot)>0$ such that the (power) normalized high-risk scenario $\left(X^{t} / t\right)^{1 / g(t)}$ converges weakly to $K$, in the sense that

$$
\mathrm{P}\left(\left(X^{t} / t\right)^{1 / g(t)}>x\right) \rightarrow \bar{K}(x) \quad \text { as } t \rightarrow x_{F}
$$

for every continuity point $x>0$ of $K$.

From the classical convergence to types theorem (see [23, Proposition 0.2]), it follows that, for $F \in D_{\mathrm{p}}^{\mathrm{POT}}(K)$, the possible limit laws $K$ are unique up to what we might call $p$-types (in the POT setting), where we call two DFs $K_{1}$ and $K_{2}$ of the same $p$-type if $K_{1}(x)=K_{2}\left(x^{p}\right)$ for some $p>0$.

In the result below we exploit the link between the two concepts of linear and power norming for high-risk scenarios. It connects the respective domains of attraction $D_{1}^{\mathrm{POT}}$ and $D_{\mathrm{p}}^{\mathrm{POT}}$.

Proposition 3.1. For $X>0$ with $D F F_{X}$ and for $\xi \in \mathbb{R}$ the following statements hold:

$$
\begin{aligned}
& \text { (i) } F_{\log X} \in D_{1}^{\mathrm{POT}}\left(H_{\xi}\right) \quad \Longleftrightarrow F_{X} \in D_{\mathrm{p}}^{\mathrm{POT}}\left(K_{\xi}\right), \\
& \text { (ii) } F_{X} \in D_{1}^{\mathrm{POT}}\left(H_{\xi}\right) \Longrightarrow F_{X} \in D_{\mathrm{p}}^{\mathrm{POT}}\left(K_{\xi_{-}}\right),
\end{aligned}
$$

where $\bar{K}_{\xi}(x)=-\log H_{\xi}(\log x)$ for $x>0$ and $\xi_{-}=\xi \wedge 0$.

Proof. (i) Let $\xi \in \mathbb{R}$ and $x>0$. Setting $Y=\log X$, the corresponding high-risk scenario satisfies $Y^{s}=\log \left(X^{t}\right)$ for $s=\log t$ and, thus, it immediately follows that

$$
\lim _{s \rightarrow x_{F}} \mathrm{P}\left(\frac{Y^{s}-s}{f(s)}>x\right)=-\log H_{\xi}(x) \Longleftrightarrow \lim _{t \rightarrow x_{F}} \mathrm{P}\left(\left(\frac{X^{t}}{t}\right)^{1 / g(t)}>x\right)=\bar{K}_{\xi}(x),
$$

where $f(s)=g(t)$ and $s=\log t$.

(ii) Let $x>0$, and assume that $F \in D_{1}^{\mathrm{POT}}\left(H_{\xi}\right)$, i.e. for some $f(\cdot)>0$ and with $x>0$,

$$
\mathrm{P}\left(\frac{X^{t}-t}{f(t)}>x\right) \rightarrow(1+\xi x)^{-1 / \xi} \quad \text { as } t \rightarrow x_{F} .
$$

We make use of the fact that the convergence above is uniformly in $t$. Moreover, define $\lambda_{t}(x)=\left(t x^{g(t)}-t\right) / f(t)$ and observe that if $\lim _{t \rightarrow x_{F}} \lambda_{t}(x)=: \lambda_{\infty}(x)$ exists, we have, for every $x>0$,

$$
\begin{aligned}
\mathrm{P}\left(\left(\frac{X^{t}}{t}\right)^{1 / g(t)}>x\right) & =\frac{\bar{F}\left(t x^{g(t)}\right)}{\bar{F}(t)} \\
& =\frac{\bar{F}\left(t+\lambda_{t}(x) f(t)\right)}{\bar{F}(t)} \\
& \rightarrow\left(1+\xi \lambda_{\infty}(x)\right)^{-1 / \xi} \text { as } t \rightarrow x_{F} .
\end{aligned}
$$

Now, set $g(t)=f(t) / t$ so that $\lambda_{t}(x)=t\left(x^{f(t) / t}-1\right) / f(t)$ for $x>0$.

Case 1: $\xi>0$. In this case $g(t) \rightarrow \xi$ as $t \rightarrow x_{F}$; see [11, Theorem 1.2.5]. Therefore, the limit $\lambda_{\infty}$ exists, is finite, and we have $\lim _{t \rightarrow x_{F}} \lambda_{t}(x)=\left(x^{\xi}-1\right) / \xi$. 
Case 2: $\xi<0$. Note first that $x_{F}<\infty$. Moreover, we have $f(t) /\left(x_{F}-t\right) \rightarrow-\xi$ as $t \rightarrow x_{F}$ (see [11, Theorem 1.2.5]), and, hence, $g(t) \rightarrow 0$ for $t \rightarrow x_{F}$. Therefore, we obtain $\lim _{t \rightarrow x_{F}} \lambda_{t}(x)=\log x$.

Case 3: $\xi=0$. For $\xi=0$, the right endpoint $x_{F}$ may be finite or infinite. Moreover, $f(\cdot)$ is asymptotically equivalent to a function $\tilde{f}(\cdot)$, whose derivative vanishes at $x_{F}$. For the case $x_{F}=\infty$, we thus have

$$
\frac{\tilde{f}(t)-\tilde{f}\left(t_{0}\right)}{t}=\frac{1}{t} \int_{t_{0}}^{t} \tilde{f}^{\prime}(s) \mathrm{d} s \rightarrow 0 \quad \text { as } t \rightarrow \infty .
$$

Therefore, $\tilde{f}(t) / t \rightarrow 0$ as $t \rightarrow x_{F}$ (and, hence, also $g(t) \rightarrow 0$ ), which in turn implies that $\lim _{t \rightarrow x_{F}} \lambda_{t}(x)=\log x$.

In the case $x_{F}<\infty, \tilde{f}(t) \rightarrow 0$ as $t \rightarrow x_{F}$ (and, hence, also $g(t) \rightarrow 0$ ); see [11, Theorem 1.2.6]. Therefore, we obtain $\lim _{t \rightarrow x_{F}} \lambda_{t}(x)=\log x$.

Altogether, $F \in D_{1}^{\mathrm{POT}}\left(H_{\xi}\right)$ with $\xi \in \mathbb{R}$ implies that, for every $x>0$ and as $t \rightarrow x_{F}$,

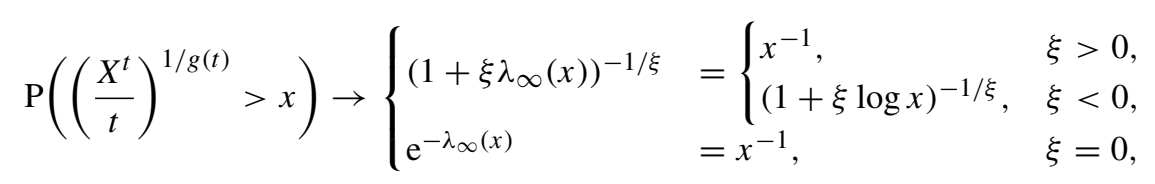

i.e. $F \in D_{\mathrm{p}}^{\mathrm{POT}}\left(K_{\xi_{-}}\right)$, where $\xi_{-}=\xi \wedge 0$. This completes the proof.

As we subsequently prefer to work within a quantile setting, a reformulation of Proposition 3.1 in terms of quantile functions is useful. Owing to the convergence properties of inverse functions (see [23, Proposition 0.1]), this is immediate and we have the following result.

Corollary 3.1. For $X>0$ with tail quantile function $U_{X}$ and $\xi \in \mathbb{R}$, the following statements hold:

$$
\begin{aligned}
& \text { (i) } U_{\log X} \in \operatorname{ERV}_{\xi}(a) \Longleftrightarrow \log U_{X} \in \operatorname{ERV}_{\xi}(a), \\
& \text { (ii) } U_{X} \in \operatorname{ERV}_{\xi}(a) \Longrightarrow \log U_{X} \in \operatorname{ERV}_{\xi-}(b),
\end{aligned}
$$

where $\xi_{-}=\xi \wedge 0$ and $b(t)=a(t) / U(t)$ for some measurable function $a(\cdot)>0$.

Remark 3.1. The respective converse implications of Proposition 3.1(ii) and Corollary 3.1(ii) do not hold; $D_{\mathrm{p}}^{\mathrm{POT}}$ attracts in fact more distributions than $D_{1}^{\mathrm{POT}}$. Consider, for example, $\bar{F}_{X}(x)=(\log x)^{-1}$ with $x>$ e; hence, $F_{X} \notin D_{1}^{\mathrm{POT}}$ but $F_{X} \in D_{\mathrm{p}}^{\mathrm{POT}}$.

\section{Second-order asymptotics of normalized quantiles}

The results below are expressed in terms of quantiles $U$ rather than distribution tails $\bar{F}$. However, any statement formulated in the $U$-framework may equivalently be expressed in the $\bar{F}$-framework. Moreover, while we worked in full generality (i.e. $\xi \in \mathbb{R}$ ) so far, we shall henceforth restrict ourselves to the case $\xi \geq 0$, of most interest for applications in insurance and finance. Similar results for the case $\xi<0$ may be worked out.

Assuming that $U \in \operatorname{ERV}_{\xi}(a)$ for some $\xi \geq 0$, i.e. for $x>0$,

$$
\frac{U(t x)-U(t)}{a(t)} \rightarrow D_{\xi}(x):=\frac{x^{\xi}-1}{\xi} \text { as } t \rightarrow \infty
$$


for some measurable function $a(\cdot)>0$, Corollary 3.1 implies that $\log U \in \Pi(b)$ and, hence,

$$
\left(\frac{U(t x)}{U(t)}\right)^{1 / b(t)} \rightarrow x \quad \text { as } t \rightarrow \infty,
$$

where $b(t)=a(t) / U(t)>0$, such that $b(t) \rightarrow \xi$. As a consequence, the (high) quantile $U(t x)$ may, for large values of $t$, either be approximated by

$$
U(t x) \approx U(t)+a(t) D_{\xi}(x)
$$

or by

$$
U(t x) \approx x^{b(t)} U(t)
$$

While the former approximation is well studied (see, for instance, [11, Section 3]), the latter is less known and, hence, of main interest in the sequel. The two approximations (4.3) and (4.4) will in general yield different results (unless $b(t) \equiv \xi$ for some $\xi>0$, in which case they coincide). In order to exploit the potential of approximation (4.4), we compare its performance with the standardly used approximation (4.3) by means of comparing the respective relative approximation errors in an asymptotic framework, followed by a simulation study in Section 5 .

Proposition 4.1. Suppose that there exist functions $b$, with $\lim _{t \rightarrow \infty} b(t)=\xi$ for some $\xi \geq 0$, and $B$, ultimately monotone and with $\lim _{t \rightarrow \infty} B(t)=0$ such that, for some $\rho \leq 0$ and $x>0$,

$$
\lim _{t \rightarrow \infty} \frac{x^{b(t)} U(t) / U(t x)-1}{B(t)}=-T_{\rho}(x),
$$

where

$$
T_{\rho}(x)= \begin{cases}\frac{1}{\rho}\left(D_{\rho}(x)-\log x\right), & \rho<0, \\ \frac{1}{2}(\log x)^{2}, & \rho=0 .\end{cases}
$$

In the case that $\xi=\rho=0$, we further assume that $\lim _{t \rightarrow \infty} B(t) /(b(t))^{2}=c \in \mathbb{R}$. Then we have, for $x>0$,

$$
\lim _{t \rightarrow \infty} \frac{\left(U(t)+a(t) D_{\xi}(x)\right) / U(t x)-1}{A(t)}=-S_{\xi, \rho}(x),
$$

where $a(t)=b(t) U(t)$,

$$
A(t)= \begin{cases}b(t)-\xi, & \rho=0<\xi \\ B(t), & \rho<0, \text { or } \xi=\rho=0 \text { and } c \neq 0, \\ (b(t))^{2}, & \xi=\rho=0, c=0\end{cases}
$$

and

$$
S_{\xi, \rho}(x)= \begin{cases}\frac{1}{\rho}\left(\log x-x^{-\xi} D_{\xi}(x)\right)+T_{\rho}(x), & \rho<0, \\ \log x-x^{-\xi} D_{\xi}(x), & \rho=0<\xi, \\ \left(1+\frac{1}{c}\right) T_{0}(x), & \xi=\rho=0, c \neq 0, \\ T_{0}(x), & \xi=\rho=0, c=0 .\end{cases}
$$


Proof. First, recall that a function $U$ is said to be of second-order ERV if there exists positive functions $a$ and $A$ with $\lim _{t \rightarrow \infty} A(t)=0$ such that, for some $\xi \in \mathbb{R}$, the limit

$$
H(x)=\lim _{t \rightarrow \infty} \frac{(U(t x)-U(t)) / a(t)-\left(x^{\xi}-1\right) / \xi}{A(t)}
$$

exists for all $x>0$ and is not a multiple of $\left(x^{\xi}-1\right) / \xi$. In this case we write $U \in 2 \operatorname{ERV}_{\xi, \rho}(a, A)$, where $\rho \leq 0$ denotes the so-called second-order parameter; see, for instance, [11, Appendix B] for details.

Now, note that (4.5) can be rewritten as, for $x>0$,

$$
\lim _{t \rightarrow \infty} \frac{-\left(x^{b(t)} U(t) / U(t x)-1\right)}{B(t)}=\lim _{t \rightarrow \infty} \frac{\log U(t x)-\log U(t)-b(t) \log x}{B(t)}=T_{\rho}(x) .
$$

Since $\lim _{t \rightarrow \infty} B(t) / b(t)=0$, we thus have $\log U \in 2 \operatorname{ERV}_{0, \rho}(b, B / b)$. The assumed form of the limit $T_{\rho}(x)$ implies that $b \in \operatorname{ERV}_{\rho}(B)$ and, hence, also that $(b(t)-\xi) \in \operatorname{ERV}_{\rho}(B)$. Moreover, we have

$$
\lim _{t \rightarrow \infty} \frac{B(t)}{b(t)-\xi}=\rho, \quad \rho \leq 0 \leq \xi
$$

see [11, Theorem B.2.2 and Corollary B.2.13].

In the case $\xi>0$, observe that, as $t \rightarrow \infty$,

$$
\begin{aligned}
-\left(\frac{U(t)+a(t) D_{\xi}(x)}{U(t x)}-1\right) \sim & \frac{U(t x)}{U(t)\left(1+b(t) D_{\xi}(x)\right)}-1 \\
\sim & x^{-\xi}\left(\frac{U(t x)}{U(t)}-1-b(t) D_{\xi}(x)\right) \\
= & \frac{U(t x)}{x^{\xi} U(t)}-1-(b(t)-\xi) x^{-\xi} D_{\xi}(x) \\
= & (\log U(t x)-\log U(t)-\xi \log x)(1+o(1)) \\
& -(b(t)-\xi) x^{-\xi} D_{\xi}(x) \\
= & b(t) \log x+T_{\rho}(x) B(t)-\xi \log x-(b(t)-\xi) x^{-\xi} D_{\xi}(x) \\
& +o(B(t))+o(b(t)-\xi) \\
= & \left(\log x-x^{-\xi} D_{\xi}(x)\right)(b(t)-\xi)+T_{\rho}(x) B(t) \\
& +o(B(t))+o(b(t)-\xi),
\end{aligned}
$$

where we have used a Taylor expansion for $\exp (\cdot)$ and (4.6). Therefore, we have

$$
\begin{aligned}
\frac{U(t)\left(1+b(t) D_{\xi}(x)\right) / U(t x)-1}{A(t)} \sim & -\left(\log x-x^{-\xi} D_{\xi}(x)\right) \frac{b(t)-\xi}{A(t)}-T_{\rho}(x) \frac{B(t)}{A(t)} \\
& +(o(B(t))+o(b(t)-\xi)) \frac{1}{A(t)}
\end{aligned}
$$

so the result follows in view of (4.7). 
Now let $\xi=0$ and recall that $\lim _{t \rightarrow \infty} B(t) / b(t)=0$. On the other hand, $b \in \operatorname{ERV}_{\rho}(B)$ implies that $B(t) / b(t) \rightarrow \rho$ and, therefore, the case $\xi=0$ necessitates $\rho=0$. Then, as $t \rightarrow \infty$,

$$
\begin{aligned}
-\left(\frac{U(t)\left(1+b(t) D_{\xi}(x)\right)}{U(t x)}-1\right) \sim & \log U(t x)-\log U(t)-\log (1+b(t) \log x) \\
= & b(t) \log x+T_{0}(x) B(t)+o(B(t)) \\
& -\left(b(t) \log x-\frac{1}{2}(\log x)^{2}(b(t))^{2}+o\left((b(t))^{2}\right)\right),
\end{aligned}
$$

and, hence,

$$
\begin{aligned}
\frac{\left(U(t)\left(1+b(t) D_{\xi}(x)\right) / U(t x)-1\right)}{A(t)} \sim & -T_{0}(x) \frac{B(t)}{A(t)}-\frac{1}{2}(\log x)^{2} \frac{(b(t))^{2}}{A(t)} \\
& +\frac{o(B(t))+o\left((b(t))^{2}\right)}{A(t)}
\end{aligned}
$$

which completes the proof.

Remarks 4.1. (i) From Proposition 4.1 and its proof, we may conclude that the (less known) approximation (4.4) performs asymptotically at least as well as approximation (4.3). Indeed, in the case $\rho<0$ the approximation errors tend to 0 at the same rate $B(t)$ (except for the special case $-\rho=\xi>0$ for which $S_{\xi,-\xi}(x) \equiv 0$ or if $\left.c=-1\right)$. In the case $\rho=0$, the error rate in (4.4) tends to 0 faster than in (4.3), except for $\rho=\xi=0 \neq c$, in which case both rates are the same. The potential improvement in the convergence rate in cases where $\rho=0$ is of particular interest because of the possible applications to quantitative risk management, where frequently used models (E. Balta, OCC, personal communication) include, for instance, the lognormal ( $\xi=\rho=0)$, the loggamma, or the g-and-h (both $\rho=0)$.

(ii) In cases where the relative approximation error of (4.4) vanishes faster than that of (4.3), the gain is not spectacular since these cases necessitate $\rho=0$. The corresponding convergence rate $B(t)$ in (4.5) is slowly varying and, thus, may tend to 0 arbitrarily slow. Similar conclusions are found in [16] in the context of penultimate approximations in the block-maxima setting.

(iii) From a methodological viewpoint, Proposition 4.1 may be seen as a partial converse of Lemma B.3.16 of de Haan and Ferreira [11]. While these authors showed how the assumption of $U \in 2 \mathrm{ERV}_{\xi, \rho}$ implies a second-order condition for $\log U$, we basically assume that $\log U \in$ $2 \mathrm{ERV}_{0, \rho}$ and analyze the implications on the second-order behavior of $U$. Note that in their framework the case $\xi=\rho$ (e.g. lognormal) is not treated. Also, in the case $\rho=0$ (e.g. loggamma and g-and-h) no nondegenerate second-order result for $\log U$ is obtained.

In summary, while Proposition 4.1 highlights the potential usefulness of approximation (4.4), the findings are asymptotic and, hence, do not guarantee a good performance for finite samples. Therefore, numerical simulations are needed in order to evaluate the potential of (4.4) for practical applications. To this end, we must first identify candidates $b(\cdot)$ satisfying (4.5). Below we consider two different choices of $b(\cdot)$ and derive sufficient conditions for (4.5) to hold.

\subsection{Sufficient conditions}

In order to avoid unnecessary technicalities and to exclude pathological cases, we will assume throughout sufficient smoothness for $U$. For our purposes, the following representation for $U$ 
turns out to be convenient to work with:

$$
U(t)=\mathrm{e}^{\varphi(\log t)}, \quad \varphi(t)=\int_{1}^{\mathrm{e}^{t}} \frac{\mathrm{d} s}{u(s)}+c .
$$

Here $u(s)=U(s) / U^{\prime}(s)$ and $c=\log U(1)$. Furthermore, we assume that

(A1) the von Mises condition holds, i.e. $t U^{\prime \prime}(t) / U^{\prime}(t) \rightarrow \xi-1$ for some $\xi \geq 0$; see [11] for details.

Assumption (A1) is equivalent to assuming that $\varphi^{\prime} \rightarrow \xi \geq 0$ together with $\varphi^{\prime \prime} / \varphi^{\prime} \rightarrow 0$. It reflects the fact that the log-log plot $\varphi$ of $U$ is assumed to behave 'nicely' in the sense of being ultimately linear, i.e. with converging slope $\varphi^{\prime}$ and vanishing convexity $\varphi^{\prime \prime}$. With this notation introduced, we have the following result on sufficient conditions for Proposition 4.1 to hold.

Proposition 4.2. Suppose that $U(t)=\mathrm{e}^{\varphi(\log t)}$ is three times differentiable and satisfies $(A 1)$.

(i) Let $b_{1}(t)=\varphi^{\prime}(\log t)$, and assume that $b_{1}^{\prime}$ is ultimately monotone and that

$$
\lim _{t \rightarrow \infty} \frac{\varphi^{\prime \prime \prime}(t)}{\varphi^{\prime \prime}(t)}=\rho
$$

for some $\rho \leq 0$. Then (4.5) holds with $b(t)=b_{1}(t)$ and $B(t)=t b_{1}^{\prime}(t)=\varphi^{\prime \prime}(\log t)$.

(ii) Let $b_{2}(t)=\log U(t)-1 / t \int_{t_{0}}^{t} \log U(s) \mathrm{d} s$ for some $t_{0}>0$, and assume that $b_{2}^{\prime}$ is ultimately monotone and that $\lim _{t \rightarrow \infty} \varphi^{\prime \prime \prime}(\log t) /\left(\varphi^{\prime \prime}(\log t)-t b_{2}^{\prime}(t)\right)-1=\rho$ for some $\rho \leq 0$. If $\rho \neq-1$ then (4.5) holds with $b(t)=b_{2}(t), B(t)=t b_{2}^{\prime}(t)$, and the limit $T_{\rho}(x)+D_{\rho}(x)$.

Proof. For (i), we may rewrite (4.5) for $x>0$ and with $t \rightarrow \infty$ as

$$
\begin{aligned}
\lim _{t \rightarrow \infty} \frac{-\left(x^{b_{1}(t)} U(t) / U(t x)-1\right)}{B(t)} & =\lim _{t \rightarrow \infty} \frac{\log U(t x)-\log U(t)-b_{1}(t) \log x}{B(t)} \\
& =\int_{1}^{x} \frac{b_{1}(t s)-b_{1}(t)}{B(t)} \frac{1}{s} \mathrm{~d} s .
\end{aligned}
$$

With $b_{1}$ as given, $\varphi^{\prime \prime \prime} / \varphi^{\prime \prime} \rightarrow \rho$ is equivalent to $t b_{1}^{\prime \prime}(t) / b_{1}^{\prime}(t) \rightarrow \rho-1$ and together with the ultimate monotonicity of $b_{1}^{\prime}$ ensures that $b_{1} \in \operatorname{ERV}_{\rho}(B)$ for some $\rho \leq 0$, such that we may choose $B(t)=t b_{1}^{\prime}(t)$. While clear for the $\rho<0$ case, this follows from the monotone density theorem for $\Pi$-variation for the $\rho=0$ case; see [5, Theorem 3.6.8]. Finally, by the uniform convergence theorem for ERV (see [5, Theorem 3.1.7a]), the convergence

$$
\lim _{t \rightarrow \infty} \frac{b_{1}(t s)-b_{1}(t)}{t b_{1}^{\prime}(t)}= \begin{cases}\frac{s^{\rho}-1}{\rho}, & \rho<0, \\ \log s, & \rho=0,\end{cases}
$$

holds locally uniformly on $(0, \infty)$, completing the proof of part (i).

The proof for (ii) is similar to (i); the main steps are as follows. With $b_{2}$ as given, the assumptions guarantee that $b_{2} \in \operatorname{ERV}_{\rho}(c)$ with $c(t)=t b_{2}^{\prime}(t)$. Furthermore, using partial integration, we obtain

$$
\log U(x)=b_{2}(x)+\int_{t_{0}}^{x} \frac{b_{2}(t)}{t} \mathrm{~d} t .
$$


Therefore, again by the uniform convergence theorem for ERV, we obtain, for $x>0$ and as $t \rightarrow \infty$

$$
\begin{aligned}
-\left(\frac{U(t x) / x^{b_{2}(t)} U(t)-1}{t b_{2}^{\prime}(t)}\right) & \sim \frac{\log U(t x)-\log U(t)-b_{2}(t) \log x}{t b_{2}^{\prime}(t)} \\
& =\frac{b_{2}(t x)-b_{2}(t)}{t b_{2}^{\prime}(t)}+\int_{1}^{x} \frac{b_{2}(t s)-b_{2}(t)}{t b_{2}^{\prime}(t)} \frac{1}{s} \mathrm{~d} s \\
& \rightarrow \frac{x^{\rho}-1}{\rho}+T_{\rho}(x)
\end{aligned}
$$

which completes the proof.

The rationale behind the choice of normalization $b_{1}$ in Proposition 4.2(i) is rather intuitive once we note that $b_{1}(t)=t U^{\prime}(t) / U(t)=\varphi^{\prime}(\log t)$ is the slope of the log-log plot of $U(t)=$ $\mathrm{e}^{\varphi(\log t)}$. Under (A1), obviously, $\varphi^{\prime}(\log t) \rightarrow \varphi^{\prime}(\infty)=\xi$, and, hence, we will refer to $b(t)=$ $\varphi^{\prime}(\log t)$ as the local or penultimate tail index of the log-log plot of $U$ at points $t$ (as opposed to the ultimate tail index $\varphi^{\prime}(\infty)=\xi$ ). Furthermore, we remark that the sufficient conditions given in Proposition 4.2(i) are, under suitable smoothness and monotonicity assumptions on $U$, close to also being necessary for (4.5).

In contrast, the choice of normalization $b_{2}$ in Proposition 4.2(ii) presents a special case. We include it in this asymptotic analysis merely to present an alternative way of choosing $b(\cdot)$. This in turn will result in a different high-quantile estimator. Unlike $b_{1}$, the rationale behind $b_{2}$ does not seem to be very intuitive at first. It may be motivated by Karamata's theorem, according to which $\varphi(\log t)$ is of the same order as its average $\tilde{\varphi}(\log t):=(1 / t) \int_{t_{0}}^{t} \varphi(\log s) \mathrm{d} s$ for some $0<t_{0}<t$, i.e. $\tilde{\varphi}(\log t) / \varphi(\log t) \rightarrow 1$ as $t \rightarrow \infty$. Therefore, we may choose $b(t)=\tilde{\varphi}^{\prime}(\log t)=\varphi(\log t)-(1 / t) \int_{t_{0}}^{t} \varphi(\log s) \mathrm{d} s$ with $0<t_{0}<t$.

In view of the discussion above, we will refer to the approximation $U(t x) \approx x^{b(t)} U(t)$ for some $b(t) \rightarrow \xi \geq 0$ as the penultimate approximation (as opposed to the ultimate approximation $\left.U(t x) \approx x^{\xi} U(t)\right)$. The idea of penultimate approximations goes back to Fisher and Tippett [14]. An early discussion of penultimate approximations in the context of block maxima is found, for instance, in [7] or [16]. Nevertheless, the potential of penultimate approximations for practical applications seems to have received limited attention so far. Motivated by the asymptotic results above, below we analyze the potential of penultimate approximations for high-quantile estimation by means of a simulation study.

\section{Implications for quantitative risk management}

We discuss the relevance of power norming, or more precisely of the corresponding penultimate approximations as discussed in the previous section. In particular, we study the EVT-based estimation of high quantiles together with possible fallacies it may bring with it. We hope that, for the EVT community, our discussion will lead to further relevant research-especially for the important case $\rho=0$.

Recall the Basel II/III regulatory guidelines for CR and OR according to which risk capital has to be calculated using VaR (i.e. quantiles) at the high level of $99.9 \%$. Owing to the nature of the problem, the use of EVT has emerged naturally; see [20] in the case of OR and [6] for CR. However, accurate estimation of the tail index $\xi$ is challenging, so some constructive scepticism concerning the wiseness to base risk capital on high-level quantiles of some (profit and) loss DF, even when using standard EVT methods, is still called for; see, for instance, [9] and [21]. 
The asymptotic results discussed in Section 4 suggest that moving away from the tail index $\xi$ - the indicator of the ultimate heavy-tailedness of the loss model-and focusing instead on the local tail index $b(t)=\varphi^{\prime}(\log t)$ or on $b(t)=\tilde{\varphi}^{\prime}(\log t)$, might prove useful at this point. In particular, it motivates the consideration and comparison of estimation methods for high quantiles based on what we would like to call (i) standard EVT, and (ii) advanced EVT (see below for more details on this nomenclature).

As for (i), we incorporate two methods belonging to the standard EVT toolkit. Recall from the asymptotics for quantiles under linear norming (see relation (4.1)) that we may consider $U(t x) \approx U(t)+a(t)\left(x^{\xi}-1\right) / \xi$ and, owing to the regular variation of $U$, also $U(t x) \approx x^{\xi} U(t)$ for $x>1$ and large values of $t$. This suggests the following scaling properties of high-quantile estimators. For some quantile levels $\tilde{\alpha}, \alpha \in(0,1)$ with $\tilde{\alpha}<\alpha$,

$$
\widehat{\operatorname{VaR}_{\alpha}}=\widehat{\operatorname{VaR}_{\tilde{\alpha}}}+\widehat{a(t)} \frac{x^{\widehat{\xi}}-1}{\widehat{\xi}},
$$

and, similarly,

$$
\widehat{\operatorname{VaR}_{\alpha}}=x^{\widehat{\xi}} \widehat{\operatorname{VaR}_{\tilde{\alpha}}}
$$

with $x=(1-\tilde{\alpha}) /(1-\alpha)>1$ and some estimates of $\xi, a(t)$, and $\operatorname{VaR}_{\tilde{\alpha}}$ at the lower level $\tilde{\alpha}$.

Relation (5.1) is better known as the POT estimator of $\operatorname{VaR}_{\alpha}$. Indeed, setting $u=\widehat{\operatorname{VaR}_{\tilde{\alpha}}}$, and using Proposition 2.1, we arrive at a natural estimator

$$
\widehat{\operatorname{VaR}_{\alpha}}=u+\widehat{f(u)} \frac{\left(N_{u} / n(1-\alpha)\right)^{\widehat{\xi}}-1}{\widehat{\xi}}
$$

for some estimates $\widehat{\xi}$ and $\widehat{f(u)}$ of $\xi$ and of $f(u)$. Here $N_{u} / n$ is an estimate of $\bar{F}(u)$, where $N_{u}$ denotes the number of exceedances over the threshold $u$ (set by the user) of a total number of $n$ data points; see, for instance, [13, Chapter 6.5].

In the simulation study below, (5.3) and (5.2) are referred to as the standard EVT I and II methods, respectively. The tail index $\xi$ and (threshold-dependent) scale parameter $f(u)$ are estimated using the POT maximum likelihood estimator method (POT-MLE method) with an ad hoc threshold choice of $10 \%$ for upper order statistics; extensive simulations (V. ChavezDemoulin, personal communication) have shown that this is an overall good first threshold choice. Compared to the POT-MLE, the performance of other implemented tail index estimators such as the Hill, the method of moments, and the exponential regression model (see, for instance, [4]) did not show significant differences.

The so-called advanced EVT approach (ii) makes use of penultimate approximations. Based on relation (4.2), with a nonconstant power normalization $b(\cdot)$, we suggest the following scaling procedure for high-quantile estimators. For quantile levels $\tilde{\alpha}, \alpha \in(0,1)$ with $\tilde{\alpha}<\alpha$,

$$
\widehat{\operatorname{VaR}_{\alpha}}=x^{\widehat{b(t)}} \widehat{\operatorname{VaR}_{\tilde{\alpha}}} \text {, }
$$

with $t=1 /(1-\tilde{\alpha}), x=(1-\tilde{\alpha}) /(1-\alpha)>1$, and some estimates of $b(t)$ and $\operatorname{VaR}_{\tilde{\alpha}}$. For the simulation study, we incorporate the two choices $b(t)=\varphi^{\prime}(\log t)$ as well as $b(t)=\tilde{\varphi}^{\prime}(\log t)$ and will refer to these methods as the advanced EVT I and II methods, respectively.

The advanced EVT methods are included in the simulation study in order to outline the potential of penultimate approximations for practical applications. For the aim of this paper, we do not elaborate on the respective estimation procedures for $\varphi^{\prime}$ and $\tilde{\varphi}^{\prime}$. In both cases, the estimates are based on a prior local regression procedure for the log data. This is done with the 'locfit' function (with a tricube weight function and smoothing parameter of $\frac{3}{4}$ ) provided in S-Plus (see [18, Chapter 3 and Section 6.1]). The integral appearing in $\tilde{\varphi}^{\prime}$ is approximated by a 
composite trapezoidal rule. Finally, the (lower) quantile $\operatorname{VaR}_{\tilde{\alpha}}$ for (5.2) and (5.4) is estimated by the empirical quantile.

Remark 5.1. (Local tail index.) The two scaling procedures (5.2) and (5.4) use the idea of a linear extrapolation of the $\log -\log$ plot $\varphi$ of $U$, but with slopes $\varphi^{\prime}$ at different quantile levels. While the penultimate approximation (5.4) requires the estimation of the local tail index $\varphi^{\prime}(\log t)$ (or of $\tilde{\varphi}^{\prime}(\log t)$ ) at a specified level $t$, the ultimate approximation (5.2)—in theory-makes use of estimates of the ultimate tail index $\varphi^{\prime}(\infty)=\xi$.

In practice, given a sample of size 1000 , say, a number of largest order statistics (above a certain threshold $t_{0}$ ) are used to estimate $\xi$ in (5.2). It is clear that this yields an estimate of $\varphi^{\prime}(\log u)$ at some (unknown) level $u>t_{0}$ rather than of $\xi=\varphi^{\prime}(\infty)$. One of the differences between (5.2) and (5.4) is thus that in the former case the level $u$ is random ( $u$ depends on the underlying data), while the latter case uses estimates of the slope $\varphi^{\prime}(\log t)$ at predefined levels $t=1 /(1-\tilde{\alpha})$, set by the user.

\subsection{Simulation study}

The simulation study is based on sample data from six frequently used OR loss models, such as the loggamma, the lognormal, the g-and-h, the Pareto, the Burr, and the generalized beta distribution of the second kind (GB2). For convenience, we recall the definition of a g-and-h $\mathrm{RV} X$ which is obtained from a standard normal RV $Z$ through

$$
X=a+b \frac{\mathrm{e}^{g Z}-1}{g} \mathrm{e}^{h Z^{2} / 2},
$$

with parameters $a, g, h \in \mathbb{R}$ and $b \neq 0$. Note that in the case $h=0$ we obtain a (shifted) lognormal RV. For the Pareto DF, we use the parameterization $\bar{F}(x)=\left(x / x_{0}\right)^{-1 / \xi}$ for $x>$ $x_{0}>0$ and some $\xi>0$. The GB2 is parameterized as in [17, p. 184], while the remaining three loss models are as in [13, p. 35].

For the results of Table 1, we simulated 200 samples of 1000 observations from each of the six loss models. For each of the four abovementioned EVT-based estimation methods, we then calculated estimates $\left(\widehat{q}_{0.999}^{(i)}\right)_{1 \leq i \leq 200}$ of VaR at level $99.9 \%$ and compared the respective bias and the standardized root mean square error (SRMSE), which is defined as

$$
\frac{1}{q_{0.999}} \sqrt{\frac{1}{200} \sum_{i=1}^{200}\left(\widehat{q}_{0.999}^{(i)}-q_{0.999}\right)^{2}} .
$$

Several simulations with different choices (for risk management practice relevance) of parameter values were performed, all of them showing a similar pattern concerning the performance of the different estimation methods; see Table 1.

Remark 5.2. Despite its inconsistency with the well-known stylized facts of OR data (power tail, i.e. $\xi>0$ ), the lognormal distribution (semi heavy tailed, i.e. $\xi=0$ ) is widely used in OR practice as a loss severity model. We include it in our simulation study primarily to question its omnipresence by highlighting some of the problems its use may bring with it.

As mentioned above, estimation at very high quantile levels by means of fitting a parametric loss model may be hard to justify. For illustrative purposes, we nevertheless perform a simulation for the six resulting parametric high-quantile estimators, based on the same data sample. An excerpt of these (expectedly) disappointing results is given in Table 2. Here, the 
TABLE 1: Bias and SRMSE (in \%) of four EVT-based estimators for VaR at the $99.9 \%$ level based on 200 datasets of 1000 observations of six different loss models.

\begin{tabular}{|c|c|c|c|c|c|c|}
\hline \multirow[b]{2}{*}{ Loss model } & Bias & SRMSE & Bias & SRMSE & Bias & SRMSE \\
\hline & \multicolumn{2}{|c|}{$\begin{array}{c}\text { Loggamma } \\
(\alpha=1.75, \beta=2)\end{array}$} & \multicolumn{2}{|c|}{$\begin{array}{c}\text { Lognormal } \\
(\mu=3.5, \sigma=1.25)\end{array}$} & \multicolumn{2}{|c|}{$\begin{array}{c}\text { g-and-h } \\
(a=b=3, \\
g=0.8, h=0.4)\end{array}$} \\
\hline Standard EVT I (POT) & 8.41 & 52.88 & 5.20 & 32.93 & 9.65 & 57.63 \\
\hline Standard EVT II $(\tilde{\alpha}=0.99)$ & 5.26 & 56.53 & -8.88 & 39.24 & 4.97 & 62.62 \\
\hline Advanced EVT I ( $\tilde{\alpha}=0.99)$ & 5.69 & 35.51 & 14.34 & 35.23 & 7.77 & 44.80 \\
\hline \multirow[t]{2}{*}{ Advanced EVT II $(\tilde{\alpha}=0.99)$} & 7.60 & 36.84 & 42.44 & 53.21 & 9.53 & 44.36 \\
\hline & \multicolumn{2}{|c|}{$\begin{array}{c}\text { Pareto } \\
\left(x_{0}=1.2\right. \\
\xi=0.75)\end{array}$} & \multicolumn{2}{|c|}{$\begin{array}{c}\text { Burr } \\
(\alpha=1, \kappa=2, \\
\tau=1.5)\end{array}$} & \multicolumn{2}{|c|}{$\begin{array}{c}\mathrm{GB} 2 \\
(a=b=2 \\
p=1.5, q=0.75)\end{array}$} \\
\hline Standard EVT I (POT) & 13.73 & 62.73 & 7.79 & 54.12 & 1.20 & 45.80 \\
\hline Standard EVT II $(\tilde{\alpha}=0.99)$ & 13.99 & 72.48 & 6.10 & 62.20 & 0.21 & 51.65 \\
\hline Advanced EVT I ( $\tilde{\alpha}=0.99)$ & -9.53 & 28.29 & 1.98 & 41.34 & -5.10 & 29.94 \\
\hline Advanced EVT II $(\tilde{\alpha}=0.99)$ & 2.66 & 41.95 & 3.60 & 39.80 & -1.69 & 32.35 \\
\hline
\end{tabular}

TABLE 2: Bias and SRMSE (in \%) of parametric estimators for VaR at the 99.9\% level based on 200 datasets of 1000 observations of three different loss models.

\begin{tabular}{lcccccc}
\hline & Bias & SRMSE & Bias & SRMSE & Bias & SRMSE \\
\cline { 2 - 7 } Loss model & \multicolumn{2}{c}{ Lognormal } & \multicolumn{2}{c}{ Burr } & \multicolumn{2}{c}{$\begin{array}{c}\text { GB2 } \\
\end{array}$} \\
\cline { 2 - 7 } & \multicolumn{2}{c}{$(\mu=3.5, \sigma=1.25$} & \multicolumn{2}{c}{$(\alpha=1, \kappa=2, \tau=1.5)$} & $p=1.5, q=0.75)$ \\
\hline Loggamma & 703.51 & 735.81 & 188.78 & 200.70 & 72.59 & 81.21 \\
Lognormal & 0.50 & 9.38 & -57.86 & 58.08 & -74.88 & 74.92 \\
g-and-h & -4.27 & 15.57 & -45.33 & 47.59 & -45.46 & 47.03 \\
Pareto & $1.04 \times 10^{13}$ & $8.51 \times 10^{13}$ & $7.87 \times 10^{19}$ & $1.029 \times 10^{21}$ & $2.57 \times 10^{10}$ & $2.33 \times 10^{11}$ \\
Burr & -89.77 & 89.81 & 1.69 & 26.73 & 20.12 & 34.35 \\
GB2 & 91.42 & 300.91 & 1.26 & 32.09 & -2.00 & 25.36 \\
\hline
\end{tabular}

model parameters are estimated using MLE, except for the g-and-h distribution, for which there is no agreed standard estimation method so far. For that case, we adapt a method suggested by Tukey [24] based on $\log _{2} n$ so-called letter values, where $n$ is the sample size.

A comparison of the results in Tables 1 and 2 clearly shows that the estimation of high quantiles based on fitting parametric models may indeed be problematic. The model uncertainty involved may be considerable (large fluctuation of the estimation errors). Moreover, from a QRM regulatory point of view, a large negative bias (i.e. underestimation of risk capital) is to be avoided. Not surprisingly, the lognormal parametric model underestimates risk capital charges considerably. While intolerable from a sound regulatory perspective, this at the same time may explain the 'attractiveness' of its use for a financial institution.

On the other hand, given the high level of $99.9 \%$, the performance of all four EVT-based methods is promising; see Table 1. A comparison within the EVT-based methods does not yield a clear ranking. However, the advanced EVT methods seem to work at least as well as the standard EVT methods, in particular exhibiting smaller SRMSE. This finding is not by accident. 
TABLE 3: Bias and SRMSE (in \%) of four EVT-based estimators for VaR at the $99.97 \%$ level based on 200 datasets of 1000,500 , and 250 observations.

\begin{tabular}{|c|c|c|c|c|c|c|}
\hline \multirow{2}{*}{ Loss model } & \multicolumn{2}{|c|}{$n=1000, \tilde{\alpha}=0.99$} & \multicolumn{2}{|c|}{$n=500, \tilde{\alpha}=0.98$} & \multicolumn{2}{|c|}{$n=250, \tilde{\alpha}=0.96$} \\
\hline & Bias & SRMSE & Bias & SRMSE & Bias & SRMSE \\
\hline \multicolumn{7}{|c|}{$\operatorname{Loggamma}(\alpha=1.25, \beta=1.25)$} \\
\hline Standard EVT I (POT) & 39.47 & 159.44 & 81.57 & 265.64 & 839.68 & 8934.55 \\
\hline Standard EVT II & 38.19 & 160.53 & 82.15 & 277.51 & 1150.21 & 11944.19 \\
\hline Advanced EVT I & -2.99 & 46.88 & -3.93 & 54.19 & -7.73 & 65.91 \\
\hline Advanced EVT II & 7.49 & 68.89 & 1.94 & 65.52 & -14.11 & 80.61 \\
\hline \multicolumn{7}{|c|}{ g-and-h $(a=b=1.5, g=0.8, h=0.6)$} \\
\hline Standard EVT I (POT) & 43.06 & 149.69 & 80.63 & 251.15 & 257.08 & 963.06 \\
\hline Standard EVT II & 39.94 & 163.40 & 84.14 & 278.85 & 362.78 & 1426.99 \\
\hline Advanced EVT I & 7.76 & 60.52 & 16.76 & 75.44 & 40.31 & 130.65 \\
\hline Advanced EVT II & 17.52 & 83.57 & 18.38 & 92.22 & 8.62 & 121.65 \\
\hline \multicolumn{7}{|c|}{ Pareto $\left(x_{0}=1, \xi=0.85\right)$} \\
\hline Standard EVT I (POT) & 33.31 & 120.47 & 105.22 & 317.70 & 176.93 & 1112.75 \\
\hline Standard EVT II & 35.14 & 135.80 & 118.95 & 354.66 & 265.77 & 1734.51 \\
\hline Advanced EVT I & -16.29 & 35.67 & -29.95 & 43.54 & -31.36 & 53.36 \\
\hline Advanced EVT II & 5.46 & 63.49 & -8.24 & 71.91 & -22.20 & 65.45 \\
\hline \multicolumn{7}{|c|}{$\operatorname{Burr}(\alpha=1, \kappa=1.5, \tau=1.25)$} \\
\hline Standard EVT I (POT) & 29.94 & 159.70 & 68.72 & 263.39 & 244.88 & 1474.04 \\
\hline Standard EVT II & 27.77 & 166.73 & 68.98 & 285.69 & 287.82 & 1566.36 \\
\hline Advanced EVT I & 5.29 & 69.86 & 24.87 & 88.72 & 81.04 & 207.97 \\
\hline Advanced EVT II & 9.26 & 75.01 & 16.09 & 79.27 & 19.82 & 99.54 \\
\hline \multicolumn{7}{|c|}{$\mathrm{GB} 2(a=1, b=2, p=1.5, q=1.25)$} \\
\hline Standard EVT I (POT) & 12.93 & 88.16 & 104.19 & 589.04 & 143.92 & 613.16 \\
\hline Standard EVT II & 11.63 & 93.63 & 108.70 & 661.79 & 207.61 & 970.47 \\
\hline Advanced EVT I & 6.58 & 58.63 & 29.20 & 97.35 & 95.53 & 245.15 \\
\hline Advanced EVT II & 12.96 & 59.20 & 24.79 & 81.35 & 49.89 & 144.99 \\
\hline
\end{tabular}

Recall that the estimation of $\varphi^{\prime}$ and $\tilde{\varphi}^{\prime}$ in the advanced EVT I and II methods is based on a local regression procedure (i.e. smoothing) of the log data. As a consequence, the estimates are more robust, which leads to smaller SRMSE values. For smaller sample sizes, we expect this behavior to become even more pronounced.

To confirm the above findings on EVT-based high-quantile estimators, we perform a second, similar study and estimate quantiles at the even more extreme level of $99.97 \%$, relevant for the calculation of so-called economic capital; see, for instance, [8, Chapter 15]. Because of Remark 5.2 we leave out the lognormal data sample. We again simulated 200 samples of 1000 , 500 , and 250 observations of very heavy-tailed data in Table 3.

From Table 3 we may draw the following conclusions. Most importantly, the potential of an advanced EVT approach to estimate extreme quantiles in the presence of very heavy tails and small sample sizes is clearly revealed. The performances of the advanced EVT I and II methods are far superior to the two standard EVT approaches. This confirms that using 
penultimate approximations instead of ultimate approximations may indeed be promising in certain situations relevant for practice (and not only from a second-order asymptotic viewpoint). The estimation errors of the two advanced EVT methods remain comparably moderate, even for small sample sizes. For the standard EVT methods on the other hand, the estimation errors are considerable for small sample sizes. From a QRM perspective, this means that relying on high-quantile estimates based on these conventional methods may become questionable.

\section{Conclusion}

In this paper we considered EVT-based high-quantile estimators and discussed scaling properties and their influence on the estimation accuracy at very high quantile levels. The scarcity of data together with the heavy-tailedness present in the data (especially for OR) turns high-quantile estimation into an inherently difficult statistical task. The nature of the problem calls for EVT in some form or other. The application of methods from the standard EVT toolkit in such applied situations is however not without problems. Our main results are as follows.

First, from a methodological perspective, it is de Haan's framework of $\Pi$-variation that is most useful for our purposes, as it allows for a unified treatment of the QRM important cases $\xi>0$ and $\xi=0$. Inherent to $\Pi$-variation is the notion of power norming (as opposed to the standardly used linear norming) of quantiles and high-risk scenarios. The use of different normalizations leads to different second-order asymptotics. It turns out that, in certain cases relevant to practice, judicious choices of a (nonconstant) power normalization-instead of a linear or a constant power normalization-may improve the rate of convergence in the respective limit results.

Second, the theory of second-order extended regular variation provides a methodological basis for the derivation of new high-quantile estimators. The application of different normalizations in the respective second-order relations translates into different scaling properties of the resulting high-quantile estimators. Our findings motivate the derivation of new estimation procedures for high quantiles by means of penultimate approximations. In particular, we proposed two advanced EVT methods which are based on the estimation of the local (pseudo) slope $\varphi^{\prime}$ (and $\tilde{\varphi}^{\prime}$ ) of the log-log plot $\varphi$ of the underlying loss model $U(t)=\mathrm{e}^{\varphi(\log t)}$. The methods proposed are intended to complement, rather than to replace, methods from the standard EVT toolkit. Their applications may be useful in situations in which the reliability of standard methods seems questionable.

Third, by means of a simulation study we showed that, in the presence of heavy tails together with data scarcity, reliable estimation at very high quantile levels, such as the $99.9 \%$ or $99.97 \%$, remains a very difficult task. Regulators as well as practitioners ought to become more aware of this issue and consequently temper their aspiration of reaching very reliable capital estimation so far in the tail of loss distributions. While our study highlights the limitations of standard EVT approaches in such cases, given the above constraint, it reveals the potential of more advanced EVT methods.

Further statistical research on advanced EVT approaches to estimate high quantiles, together with a more in-depth study of their benefits as well as limitations for practical applications, would be desirable.

\section{Acknowledgements}

The authors would like to thank Guus Balkema for useful discussions related to the paper as well as the anonymous referees for their constructive remarks. Matthias Degen acknowledges 
financial support from the Swiss National Science Foundation and thanks ORIE at Cornell University for the hospitality. As Senior SFI (Swiss Finance Institute) Professor, Paul Embrechts thanks the SFI for financial support.

\section{References}

[1] Balkema, G. And Embrechts, P. (2007). High Risk Scenarios and Extremes. A geometric approach. European Mathematical Society, Zürich.

[2] Barakat, H. M., Nigm, E. M. ANd El-Adll, M. E. (2010). Comparison between the rates of convergence of extremes under linear and under power normalization. Statist. Papers 51, 149-164.

[3] Basel Committee on Banking Supervision (2008). Guidelines for computing capital for incremental risk in the trading book. Bank for International Settlements, Basel.

[4] Beirlant, J., Goegebeur, Y., Teugels, J. And Segers, J. (2004). Statistics of Extremes. John Wiley, Chichester.

[5] Bingham, N. H., Goldie, C. M. And Teugels, J. L. (1987). Regular Variation. Cambridge University Press.

[6] Chavez-Demoulin, V. and Embrechts, P. (2011). An EVT primer for credit risk. In The Oxford Handbook of Credit Derivatives, eds A. Lipton and A. Rennie, Oxford University Press, pp. 500-532.

[7] Cohen, J. P. (1982). Convergence rates for the ultimate and penultimate approximations in extreme-value theory. Adv. Appl. Prob. 14, 833-854.

[8] Crouhy, M., Galai, D. and Mark, R. (2006). The Essentials of Risk Management. McGraw-Hill, New York.

[9] Danítelsson, J. et al. (2001). An academic response to Basel II. Financial Markets Group, London School of Economics.

[10] De HaAn, L. (1970). On Regular Variation and Its Applications to the Weak Convergence of Sample Extremes (Math. Centre Tracts 32), Mathematisch Centrum, Amsterdam.

[11] De HaAn, L. And Ferreira, A. (2006). Extreme Value Theory. Springer, New York.

[12] Embrechts, P. ANd Hofert, M. (2010). A note on generalized inverses. Preprint, ETH Zürich.

[13] Embrechts, P., Klüppelberg, C. And Mikosch, T. (1997). Modelling Extremal Events. Springer, Berlin.

[14] Fisher, R. A. AND Tippett, L. H. C. (1928). Limiting forms of the frequency distribution of the largest or smallest member of a sample. Proc. Camb. Phil. Soc. 24, 180-190.

[15] Gnedenko, B. (1943). Sur la distribution limite du terme maximum d'une série aléatoire. Ann. Math. 44, 423-453.

[16] Gomes, M. I. And de HaAn, L. (1999). Approximation by penultimate extreme value distributions. Extremes 2, $71-85$.

[17] Kleiber, C. And Kotz, S. (2003). Statistical Size Distributions in Economics and Actuarial Sciences. Wiley, Hoboken, NJ.

[18] LoAder, C. (1999). Local Regression and Likelihood. Springer, New York.

[19] Mohan, N. R. And Ravi, S. (1992). Max domains of attraction of univariate and multivariate $p$-max stable laws. Theoret. Prob. Appl. 37, 632-643.

[20] Moscadelli, M. (2004). The modelling of operational risk: experiences with the analysis of the data collected by the Basel Committee. Working Paper No 517, Bank of Italy.

[21] Nešlehová, J., Embrechts, P. And Chavez-Demoulin, V. (2006). Infinite mean models and the LDA for operational risk. J. Operat. Risk 1, 3-25.

[22] Pantcheva, E. (1985). Limit theorems for extreme order statistics under nonlinear normalization. In Stability Problems for Stochastic Models (Uzhgorod, 1984; Lecture Notes Math. 1155), Springer, Berlin, pp. 284-309.

[23] Resnick, S. I. (1987). Extreme Values, Regular Variation, and Point Processes. Springer, New York.

[24] Tukey, J. W. (1977). Exploratory Data Analysis. Addison-Wesley, Reading. 